\title{
Information Gaps for Risk and Ambiguity
}

\author{
Russell Golman, George Loewenstein, and Nikolos Gurney* \\ May 6, 2015
}

\begin{abstract}
We apply a model of preferences for information to the domain of decision making under risk and ambiguity. An uncertain prospect exposes an individual to an information gap. Gambling makes the missing information more important, attracting more attention to the information gap. To the extent that the uncertainty (or other circumstances) makes the information gap unpleasant to think about, an individual tends to be averse to risk and ambiguity. Yet when an information gap happens to be pleasant, an individual may seek gambles providing exposure to it. The model provides explanations for source preference regarding uncertainty, the comparative ignorance effect under conditions of ambiguity, aversion to compound risk, and other phenomena. We present an empirical test of one of the model's novel predictions.
\end{abstract}

KEYWORDS: ambiguity, gambling, information gap, risk, uncertainty

JEL classification code: D81

*Department of Social and Decision Sciences, Carnegie Mellon University, 5000 Forbes Ave, Pittsburgh, PA 15213, USA. E-mail: rgolman@andrew.cmu.edu; g120@andrew.cmu.edu; nmgurney@ andrew.cmu.edu 


\section{Introduction}

In this paper we derive both risk and ambiguity preferences from an underlying model of preferences for information. We argue that risk and ambiguity aversion arise from the discomfort of thinking about missing information regarding either outcomes or probabilities. Likewise, risk and ambiguity seeking occur in (rarer) cases in which thinking about the missing information is pleasurable. The main focus of our model is, therefore, on when and how people think about missing information, and the hedonic consequences of doing so.

We define an information gap (Golman and Loewenstein, 2015a) as a question that one is aware of but for which one is uncertain between possible answers, and propose that the attention paid to such an information gap depends on two key factors: salience, and importance. ${ }^{1}$ The salience of a question indicates the degree to which contextual factors in a situation highlight it. Salience might depend, for example, on whether there is an obvious counterfactual in which one does possess the missing information. The importance of a question is a measure of how much one's utility would depend on the actual answer. It is this factor - importance - which is influenced by actions like wagering on a gamble. We propose that wagering on a gamble raises the importance of the gamble's associated questions (e.g., will I win, or what is my chance of winning?), which motivates one to wager on gambles that are enjoyable to think about and to not wager on gambles that are aversive to think about. Consistent with such an account, lottery players often prefer to spread out drawings, perhaps in order to savor their thoughts about the possibility of winning (Kocher et al., 2014). Likewise, people are especially prone to insure against the loss of things they have an emotional attachment to (Hsee and Kunreuther, 2000), in our view because they find it unpleasant to think about losing these items. Similarly, financial professionals primed to think about the bust of a financial bubble become more risk averse (even with known probabilities in a laboratory setting) than those primed to think about a boom (Cohn et al., 2015). In natural settings, it has been argued, the discomfort of thinking about risky situations is perhaps the primary motive behind risk avoidance (Loewenstein et al., 2001; see also Tymula et al., 2012).

Our theoretical model is substantially different from prior theoretical work that has provided accounts of risk and ambiguity preferences that are inconsistent with expected utility

\footnotetext{
${ }^{1}$ Our application of this model to information acquisition and avoidance (Golman and Loewenstein, 2015b) assumes that a third factor - surprise - contributes to attention when information is acquired, but this assumption is unnecessary here.
} 
over prizes (behavior such as low-stakes risk aversion (Rabin, 2000), the Allais (1953) common consequence and common ratio paradoxes, and the Ellsberg (1961) paradox). These other approaches typically incorporate departures from expected utility maximization, such as loss aversion (Kahneman and Tversky, 1979), non-additive probability weighting (Quiggin, 1982), and imprecise (set-valued) probabilities (Gilboa and Schmeidler, 1989). In constrast to these other approaches, our model adheres to expected utility, albeit over beliefs rather than outcomes.

Our model is not intended to be a mutually exclusive alternative to these other theories of risk and ambiguity preference, which capture many well-established behavioral patterns (see, e.g., Tversky and Kahneman, 1992; Köszegi and Rabin, 2006; Schmidt et al., 2008; Loomes and Sugden, 1982; Bordalo et al., 2012). Incorporating features from these theories would no doubt improve the predictive power of our model. For simplicity, however, the only departure of our model from traditional expected utility is that people derive utility from beliefs in addition to outcomes. Within this framework, we are able to account for a wide range of anomalous phenomena under risk and ambiguity. No model, however, including ours, can account for the full range of anomalous patterns of risk and ambiguity preference.

In the case of risk, in our model, the key question about which people are uncertain - the information gap - centers around the eventual outcome when the uncertainty is resolved. For example, when deciding whether to accept a fair odds bet on a coin toss, the information gap is whether the coin flip will come out heads or tails. Thinking about the coin turning up heads (or about it turning up tails) does not seem intrinsically pleasurable or painful, but we suggest that the feeling of uncertainty about this outcome is a source of discomfort. Thus, in our model, risk aversion (even with low stakes) arises from a desire to avoid thinking about such uncertainty. Moreover, the model predicts, there will be stronger risk aversion when the outcome depends on additional uncertainties, so there will be more pronounced aversion to compound lotteries. Our predictions of risk aversion (rather than risk seeking) require information gaps to be unpleasant to think about. Despite the discomfort associated with feelings of uncertainty, information gaps may be pleasurable to think about if the events under consideration have intrinsically positive valence, in which case our model would predict risk seeking. We would predict risk seeking behavior, for example, by a basketball fan for a lottery determined by his favorite basketball player making a free throw.

In the case of ambiguity, an additional key question comes into play: what the likeli- 
hoods are of obtaining different outcomes. ${ }^{2}$ When thinking about this question is aversive, then we expect people to be ambiguity averse; when pleasurable, they should be ambiguity seeking. This account of ambiguity preference is related to an account proposed by Frisch and Baron (1988) according to which ambiguity aversion arises from the awareness that one is missing information that would help one to refine one's judgment of a gamble's probabilities. Our account is similar to theirs in terms of focusing on missing information as the source of ambiguity preference. However, our account is more specific about how and why thinking about the information gap leads to ambiguity preference. Our account allows for the idea that thinking about information gaps can be pleasurable, and make the prediction that in these situations people will be ambiguity seeking.

While related to Frisch and Baron's account of ambiguity preference, this account is quite different from other explanations that have been proposed. For example, Ellsberg (1961), in the paper that introduced his eponymous paradox, proposed that people are pessimistic, fearing that the unknown probabilities will end up being unfavorable. Ellsberg's account seems reasonable enough in the scenario he described, but, like Frisch and Baron's account, it fails to explain many of the observed differences in ambiguity preference across situations, or, most dramatically, to accommodate the not infrequent cases of ambiguity seeking.

Other models aim to capture ambiguity preference but shed no light on its underlying cause. These models are intended to represent ambiguity-averse (and sometimes ambiguity-seeking) preferences, but they are not meant to be explanations for these preferences - the preferences are seen as fundamental. For example, ambiguity preferences have been captured by assuming non-additive subjective probability weighting (as in Schmeidler's (1989) Choquet expected utility model), ${ }^{3}$ or imprecise (set-valued) probabilities (as in Gilboa and Schmeidler's (1989) Maxmin expected utility model or, more generally, Ghirardato et al.'s (2004) $\alpha$-maxmin model or Maccheroni et al.'s (2005) variational prefer-

\footnotetext{
${ }^{2}$ Ambiguity is sometimes taken to mean that subjective odds cannot even be formulated, but such a situation would be extreme. People make subjective probability judgments all the time. In our view, the distinction between ambiguity and risk is the decision maker's awareness (and uncertainty) about sources of uncertainty. With the so-called known urn in the Ellsberg paradox, the only uncertainty is about which ball will be drawn, and there is unawareness of the mechanism that will determine it. With the ambiguous urn, the decision maker is aware of an additional uncertainty about the contents of the urn in the first place. This makes a subjective probability judgment about the color of the drawn ball uncertain, but not impossible.

${ }^{3}$ Non-additive subjective probability weighting captures ambiguity aversion when the weights are supermodular. These weights should not be interpreted as subjective probability judgments but merely as inputs into the decision model.
} 
ences model), ${ }^{4}$ or second-order risk aversion (toward distributions of outcomes) rather than reduction of compound lotteries (as in Segal's $(1987$; 1990) extension of rank dependent utility, Klibanoff et al.'s (2005) smooth model, or other recursive expected utility models (Nau, 2006; Ergin and Gul, 2009; Seo, 2009)). In contrast, we aim to derive ambiguity aversion - and, in appropriate situations, ambiguity seeking - by considering fundamental preferences for information as well as over outcomes.

In a study that provided neural support for our interpretation of ambiguity aversion, Hsu et al. (2005) scanned the brains of subjects as they made choices involving ambiguous and unambiguous gambles. The authors found that the level of ambiguity in choices correlated positively with activation in the amygdala, a brain region that has been connected by numerous studies to the experience of fear. The authors conclude that "under uncertainty, the brain is alerted to the fact that information is missing, that choices based on the information available therefore carry more unknown (and potentially dangerous) consequences, and that cognitive and behavioral resources must be mobilized in order to seek out additional information from the environment." Additional studies have found that decision making involving ambiguous gambles, and even the perception of ambiguity in the absence of decision making, correlates with activity in the posterior inferior frontal sulcus / posterior inferior frontal gyrus (Huettel et al., 2006; Bach et al., 2009), a region of the brain that has been independently identified as responsible for attentiveness to relevant information in a task switching paradigm (Brass and von Cramon, 2004). Consistent with our information gap account, this region of the brain responds to ambiguity when information (that could potentially be known) is hidden from the observer, but not under conditions of complete ignorance (Bach et al., 2009).

In building our model on the foundation of information preference, our model can help to help to explain when and why ambiguity preference takes different forms in different situations, including those that produce ambiguity seeking rather than aversion. One line of research (Fox and Tversky, 1995) shows that people value ambiguous and unambiguous gambles with similar subjective probabilities almost identically; it is only when the two types of gambles are compared to one-another that people become averse to ambiguity. The observation that people are more ambiguity averse when making choices between

\footnotetext{
${ }^{4}$ Imprecise probability captures ambiguity aversion when the decision maker is cautious or pessimistic and considers worst-case scenarios. Yet in many real world decision environments, there is so much uncertainty that worst-case scenarios would render a decision maker impossibly conservative. Moreover, evidence suggests that people are often extremely optimistic in the face of uncertainty (Weinstein, 1980; Taylor and Brown, 1988).
} 
ambiguous and unambiguous gambles can be explained by the information gap account, assuming that such comparisons tend to raise the baseline attention weight on the information gap(s) relating to the probabilities associated with the ambiguous option.

Another line of research (Heath and Tversky, 1991) shows that people actually like to bet on ambiguous outcomes - e.g., a horse race - when they feel they are expert in the domain. People tend to be averse to ambiguity when they feel they are lacking information or expertise in a domain. The information gap account of ambiguity preference can easily account for these findings with a natural assumption that it is more pleasurable to think about issues one is more expert on. Betting in domains of expertise increases the attention weight on many questions about which one is confident, whereas betting on unfamiliar situations increases the attention weight on questions one is more uncertain about. We thus should expect people to have preferences over the source of uncertainty, generally preferring a familiar source to an unfamiliar source. In fact, people do prefer to bet on their vague beliefs in situations in which they feel especially competent or knowledgeable, but prefer to bet on chance when they do not (Heath and Tversky, 1991; Taylor, 1995; Keppe and Weber, 1995; Abdellaoui et al., 2011).

Such 'source preference' may also help explain the common observation of relative over-investment in one's domestic stock market and under-investment in foreign markets (Kilka and Weber, 2000). Note that a similar mechanism could easily account for the common phenomenon of risk-seeking observed, especially, in the domain of gambling. Gamblers often believe they have expertise on the particular events they wager on. They notoriously obey superstitions about hot or cold tables in a casino and rely on 'systems' for choosing their stakes, even though many would acknowledge that the house retains a mathematical edge.

A serious limitation for many models of decision making under risk and ambiguity is their failure to make sense of the many situations in which people seem to enjoy taking risks, including lotteries and other forms of gambling. von Neumann and Morgenstern (1944) explicitly disregarded the utility of gambling in capturing risk preferences with expected utility (see also Luce and Raiffa, 1957). Others have tried to incorporate intrinsic preferences for or against gambling into an expected utility framework (e.g., Fishburn, 1980; Diecidue et al., 2004), but they associate a cost or benefit with a specific profile of material outcomes and probabilities (i.e., a "lottery"). A realistic behavioral model of intrinsic preferences about gambling must acknowledge that such preferences depend on the situation that gives rise to the gamble. In our model, the utility or disutility of gambling 
is not attached to the risk inherent in a gamble, but instead to the source of that risk.

Like other accounts of ambiguity aversion that draw a connection between risk and ambiguity preference by assuming that ambiguity preference reflects second-order risk aversion (Segal, 1987; Klibanoff et al., 2005; Nau, 2006; Ergin and Gul, 2009; Seo, 2009), our account also proposes that both phenomena stem from the same underlying mechanism, but, as we have already described, introduces a novel mechanism involving informational preferences. We do not doubt that other mechanisms, such as utility function curvature or a precautionary principle, also play a role in risk and ambiguity preferences. Nevertheless, we, like Caplin and Leahy (2001) and Epstein (2008), believe that affective feelings about uncertainty (i.e., information gaps) critically affect risk and ambiguity preferences. We suggest that these preferences are driven, or at least influenced, by the desire to not draw attention to questions one does not like thinking about.

The most straightforward, novel prediction of our model is that people should be ambiguity seeking in situations in which they enjoy thinking about the uncertainties associated with a gamble. We test, and provide strong empirical support for, this novel prediction in a new experiment reported in the paper.

We proceed, in Section 2, to introduce our formal model. Section 3 presents results applying this model to decisions under risk and Section 4 deals with ambiguity. We report experimental results supporting our theory in Section 5. Section 6 concludes. All mathematical proofs are in the appendix.

\section{Theoretical Framework}

Following Golman and Loewenstein (2015a), we represent a person's state of awareness with a set of activated questions $\mathcal{Q}=\left\{Q_{1}, \ldots, Q_{m}\right\}$, where each question $Q_{i}$ has a set of possible (mutually exclusive) answers $\mathcal{A}_{i}=\left\{A_{i}^{1}, A_{i}^{2}, \ldots\right\}$. We let $X$ denote a set of prizes. Denote the space of answer sets together with prizes as $\alpha=\mathcal{A}_{1} \times \mathcal{A}_{2} \times \cdots \times \mathcal{A}_{m} \times X$. A cognitive state can then be defined by a probability measure $\pi$ defined over $\alpha$ (i.e., over possible answers to activated questions as well as eventual prizes) and a vector of attention weights $\mathbf{w}=\left(w_{1}, \ldots, w_{m}\right) \in \mathbb{R}_{+}^{m}$. A utility function is defined over cognitive states, written as $u(\pi, \mathbf{w})$.

The probability measure reflects a subjective probability judgment about the answers to the activated questions and the prizes that may be received. The subjective probability over these prizes is in general mutually dependent with the subjective probability over answers to activated questions. That is, material outcomes may correlate with answers about activated 
questions (and the answer to one question may correlate with the answer to another). We can consider a marginal distribution $\pi_{i}$ that specifies the subjective probability of possible answers to question $Q_{i}$ or $\pi_{X}$ that specifies the subjective probability over prizes. ${ }^{5}$

The attention weights specify how much a person is thinking about each question and, in turn, how much the beliefs about those questions directly impact utility. The attention $w_{i}$ on question $Q_{i}$ is assumed to be strictly increasing in, and to have strictly increasing differences in, the question's importance $\gamma_{i}$ and salience $\sigma_{i}$. To characterize the importance of question $Q_{i}$, we consider the probabilities of discovering any possible answer $A_{i} \in \mathcal{A}_{i}$ (or, omitting answers thought to be impossible, in the support of the individual's belief about the question, $\left.\operatorname{supp}\left(\pi_{i}\right)\right)$ and the utilities of the cognitive states $\left(\pi^{A_{i}}, \mathbf{w}^{A_{i}}\right)$ that would result from discovering each possible answer $A_{i}$. We assume that the importance $\gamma_{i}$ of question $Q_{i}$ is a function of the subjective distribution of utilities that would result from different answers to the question,

$$
\gamma_{i}=\phi\left(\left\langle\pi_{i}\left(A_{i}\right), u\left(\pi^{A_{i}}, \mathbf{w}^{A_{i}}\right)\right\rangle_{A_{i} \in \operatorname{supp}\left(\pi_{i}\right)}\right)
$$

that increases with mean-preserving spreads of the distribution of utilities and that is invariant with respect to constant shifts of utility.

We assume utility takes the form $u(\pi, \mathbf{w})=u_{X}\left(\pi_{X}\right)+\sum_{i=1}^{m} w_{i} v_{i}\left(\pi_{i}\right){ }^{6}$ The first term describes the utility of a subjective distribution over prizes and the remaining terms describe the utilities of beliefs about each activated question, amplified by the attention weights on each of these questions. We can identify as positive (neutral / negative) beliefs those for which increasing attention on the belief increases (does not affect / decreases) utility.

We further assume that the value of a belief (e.g., $v_{i}\left(\pi_{i}\right)$ ) depends only on the valences of the answers that are considered possible (e.g., $v_{i}\left(A_{i}\right)$ for all $A_{i} \in \operatorname{supp}\left(\pi_{i}\right)$ ) and the amount of uncertainty in the belief. ${ }^{7}$ Golman and Loewenstein (2015a) posit a fundamental preference for clarity, which asserts that more uncertainty in a belief decreases its value. While entropy serves as a natural measure of the uncertainty in a belief, we need not make any assumptions quantifying uncertainty for our purposes here, and we instead simply assume their one-sided sure-thing principle, which holds that people always prefer a certain answer to uncertainty amongst answers that all have valences no better than the certain an-

\footnotetext{
${ }^{5}$ For any $\tilde{\mathcal{A}} \subseteq \mathcal{A}_{i}$, we have $\pi_{i}(\tilde{\mathcal{A}})=\pi\left(\mathcal{A}_{1} \times \cdots \times \mathcal{A}_{i-1} \times \tilde{\mathcal{A}} \times \mathcal{A}_{i+1} \times \cdots \times \mathcal{A}_{m} \times X\right)$.

${ }^{6}$ Golman and Loewenstein's (2015a) separability, monotonicity, and linearity properties would imply this form for the utility function.

${ }^{7}$ This is Golman and Loewenstein's (2015a) label independence property.
} 
swer (holding attention weight constant). If for all $A_{i} \in \operatorname{supp}\left(\pi_{i}\right)$ we have $v_{i}\left(\pi_{i}^{\prime}\right) \geq v_{i}\left(A_{i}\right)$, then $v_{i}\left(\pi_{i}^{\prime}\right) \geq v_{i}\left(\pi_{i}\right)$, with this inequality strict whenever $\pi_{i}$ is not degenerate.

Finally, we assume that $u_{X}\left(\pi_{X}\right)=\sum_{x \in X} \pi_{X}(x) v_{X}(x) .{ }^{8}$ That is, apart from the utility derived from beliefs (and the attention paid to them), we would have expected utility over prizes. This assumption may well be unrealistically strong (it may preclude patterns of risk seeking for moderately likely losses or longshot gains, for example), but it simplifies the model so we can focus on the impact of beliefs on utility. Thus, to the extent that our account can reconcile phenomena that a traditional expected utility model cannot, the explanation will feature the utility of beliefs.

Wagering on an uncertain gamble is a kind of instrumental action that changes the chances of receiving various prizes, typically making them contingent on the answers to particular activated questions. The obvious effect on the cognitive state is to transform the probability measure by providing new beliefs about the distribution over prizes conditional on beliefs about activated questions. A second effect is to impact attention weights because the change in prizes affects the importance of any question on which the prize is contingent. Such an action $a$, acting on a given cognitive state $(\pi, \mathbf{w})$, determines a new cognitive state $(\pi[a], \mathbf{w}[a])$. It specifies a map from every answer set $\mathbf{A} \in \mathcal{A}_{1} \times \cdots \times \mathcal{A}_{m}$ to a conditional distribution over prizes in $\Delta(X)$. Along with the prior subjective judgment about the probability of each answer set, which is preserved by the action, this defines the new subjective probability measure $\pi[a] \in \Delta(\alpha)$. The new attention weights $\mathbf{w}[a]$ are determined by new values of importance as described by Equation (1). Preference between actions is determined by their impacts on the cognitive state, in accordance with the utility function $\mathfrak{u}(a \mid \pi, \mathbf{w})=u(\pi[a], \mathbf{w}[a])-u(\pi, \mathbf{w})$.

\section{Risk}

\subsection{Low-Stakes Risk Aversion}

People tend to be risk averse, even over low-stakes lotteries (Holt and Laury, 2002). The utility curvature needed to explain low-stakes risk aversion in a traditional expected utility model implies an absurd amount of risk aversion in high-stakes lotteries, such that, for example, an individual who at any wealth level rejects a 50-50 lottery to either gain $\$ 110$ or lose $\$ 100$ would have to reject a 50-50 lottery with a potential loss of $\$ 1000$, regardless of the potential gain (Rabin, 2000). Utility function curvature almost certainly does play

\footnotetext{
${ }^{8}$ This would follow from Golman and Loewenstein's (2015a) property of independence across prizes, which is von Neumann and Morgenstern's (1944) independence axiom restricted just to material outcomes.
} 
a role in risk aversion, but clearly something more is in play here, too. We suggest that betting on a lottery exacerbates the pain of thinking about an information gap by making it more important. ${ }^{9}$

To illustrate the information gap account for low-stakes risk aversion, consider a simplifying assumption that the value function for prizes $v_{X}$ is linear over monetary prizes. (Of course, diminishing sensitivity to larger monetary prizes would be realistic, but any differentiable value function can be well approximated by a linear function over a small neighborhood.) Consider a possible bet on a fair coin that could either pay $x^{*}$ (win) or $-x^{*}$ (lose). Assume the decision maker has no intrinsic preference for heads or for tails (apart from the preference to win the lottery, if the bet is accepted), assigning both outcomes neutral valence. Then the decision maker will strictly prefer rejecting the bet.

Proposition 1 Assume $v_{X}$ is linear over $\mathbb{R}$. Suppose question $Q_{1}$ is about the outcome of the coin toss, so that it is independent of other questions, it is believed to be a fair coin with $\pi_{1}(\mathrm{H})=\pi_{1}(\mathrm{~T})=\frac{1}{2}$, and both heads and tails have neutral valence, i.e., $v_{1}(\mathrm{H})=$ $v_{1}(\mathrm{~T})=0$. Suppose bet $b$ attaches prize $x^{*}$ to heads and $-x^{*}$ to tails, so that $\pi_{X}^{\mathrm{H}}[b]\left(x^{*}\right)=$ $\pi_{X}^{\mathrm{T}}[b]\left(-x^{*}\right)=1$. Suppose not betting $(\neg b)$ attaches prize 0 to both heads and tails, so that $\pi_{X}[\neg b](0)=1$. There is a strict preference not to bet, $\neg b \succ b$.

The intuition is that having to think about the outcome of the coin toss lowers utility, because the uncertainty is aversive. Betting on the coin toss makes it more important, and the information gap would then attract more attention.

\subsection{A Preference for Certainty}

The observed patterns of non-standard risk preferences mostly seem to relate to a preference to avoid exposure to uncertainty relative to having certainty. The preference for certainty is well documented (e.g., Callen et al., 2013) and follows naturally from the information gap account. The pain of thinking about an information gap leads to what might be called direct risk aversion, above and beyond the risk aversion that can result from utility function curvature. There is a direct cost in the utility function simply from awareness of exposure to risk (i.e., from the existence of an information gap). ${ }^{10}$ Direct risk aver-

\footnotetext{
${ }^{9}$ In a rare case in which an uncertain lottery is pleasant to think about, we would suggest that risk seeking arises from the same mechanism.

${ }^{10}$ Ambiguity involves even more awareness of uncertainty than simple risk, so the information gap account also implies that there is an even larger direct utility cost from exposure to ambiguity, assuming this additional uncertainty is unpleasant to think about. Analogous to the uncertainty effect for risk, Andreoni et al. (2014) find that many subjects evaluating compound lotteries with a component that may be ambiguous actually violate (first-order stochastic) dominance as if there is a direct cost just to considering ambiguity.
} 
sion could underlie Gneezy et al.'s (2006) uncertainty effect, in which individuals value a risky prospect (say, a lottery between gift certificates worth $\$ 50$ or $\$ 100$ ) less than its worst possible realization (i.e., a $\$ 50$ gift certificate for sure). (See also Simonsohn's (2009) replication of the uncertainty effect.) In our model, this extreme of direct risk aversion would require the uncertainty to relate to highly negative beliefs. Of course this state of affairs is rare. Given the empirical facts, we might speculate that people associate the particular task of paying for a lottery over gift certificates with the danger of being suckered into a bad deal (Yang et al., 2013), which might well be a highly negative belief (see Prelec and Loewenstein, 1998; Weaver and Frederick, 2012).

\subsection{Compound Risk Aversion}

Seeing that people generally try to avoid exposure to an information gap, we might expect that compound lotteries - which expose an individual to multiple information gaps - are even more aversive. Indeed, the empirical evidence is clear that people do not reduce compound lotteries, or at least do not value them equivalently to their reduced form versions (Bernasconi and Loomes, 1992; Halevy, 2007; Abdellaoui et al., 2013; Spears, 2013). This phenomena is particularly challenging to capture with theories that do not allow for framing effects and that require the utility of a lottery to depend only on the possible outcomes and their probabilities. It is also a necessary consequence of the information gap account.

In this model, as long as the lotteries do not involve events with positive intrinsic valence, a compound lottery will be less preferred than an equivalent simple lottery.

Proposition 2 Suppose a subset of questions $\mathcal{Q}^{E} \subset \mathcal{Q}$ is believed to be independent of other questions and to have answers with neutral valence so that a belief in any such answer with certainty is a neutral belief. ${ }^{11}$ Suppose questions $Q_{i}$ and $Q_{j} \in \mathcal{Q}^{E}$ both have the same salience $\left(\sigma_{i}=\sigma_{j}\right)$ and are viewed to have the same subjective probabilities $\left(\pi_{i}\left(A_{i}^{h}\right)=\pi_{j}\left(A_{j}^{h}\right)\right)$, but the belief about question $Q_{i}$ is pairwise dependent with belief about some question $Q_{\tilde{\imath}} \in \mathcal{Q}^{E} \backslash\left\{Q_{i}, Q_{j}\right\}$ whereas the belief about question $Q_{j}$ is independent. Given a sequence of prizes $x_{h} \in X$ with distinct valences, $v_{X}\left(x_{h_{1}}\right) \neq v_{X}\left(x_{h_{2}}\right)$ for $h_{1} \neq$ $h_{2}$, consider a pair of actions $a_{i}$ and $a_{j}$ that attach prize $x_{h}$ to answer $A_{\nu}^{h}$ of question $Q_{\nu}$, $\nu \in\{i, j\}$, so that $\pi_{X}^{A_{\nu}^{h}}\left[a_{\nu}\right]\left(x_{h}\right)=1$ for all $h$. Any such bet attached to question $Q_{j}$ would be preferred to the same bet attached to question $Q_{i}$, i.e., $a_{j} \succ a_{i}$.

The intuition here is that the compound lottery (in contrast to the simple lottery) exposes the decision maker to additional information gaps. By assumption, these information gaps

\footnotetext{
${ }^{11}$ That is, $v_{i}\left(A_{i}\right)=0$ for all $A_{i} \in \mathcal{A}_{i}$, for $Q_{i} \in \mathcal{Q}^{E}$.
} 
are unpleasant to think about. Putting a prize on the line to depend on the outcome of the uncertain events makes these information gaps more important. That makes the compound lottery worse than the simple lottery.

\section{Ambiguity}

Information gaps underlie ambiguity as well as risk. Consider the preference for the known urn in Ellsberg's problem. Even if you bet on the urn with the known proportions of balls, the proportion of balls in the other urn you could have selected is still a piece of missing information. To explain the phenomenon in terms of our model, therefore, we need to assume that there is a relevant question for both urns: "What is the proportion of each colored ball?" and that the attention weight is relatively greater for the question relating to the urn you choose. This follows from the assumption that attention weight increases in a question's importance. One knows the answer to the question for the precisely specified urn, but not for the ambiguous one.

\subsection{Ellsberg Two-Urn Paradox}

In the Ellsberg two-urn paradox, subjects are presented with 2 urns. Urn I contains 100 red and black balls, but in an unknown ratio. Urn II has exactly 50 red and 50 black balls. Subjects must choose an urn to draw from, and bet on the color that will be drawn - they will receive a $\$ 100$ payoff if that color is drawn, and $\$ 0$ if the other color is drawn. Subjects must decide which they would rather bet on: 1) A red draw from Urn I, or a black draw from Urn I; 2) A red draw from Urn II, or a black draw from Urn II; 3) A red draw from Urn I, or a red draw from Urn II; and 4) A black draw from Urn I, or a black draw from Urn II. Intuition suggests that people will be indifferent between red and black in choices 1 and 2, by the principle of insufficient reason, but will prefer Urn II to Urn I in choices 3 and 4 because this urn is less ambiguous. The axioms of subjective expected utility theory (Savage, 1954 or Anscombe and Aumann, 1963), however, imply that a preference for an urn in choice 3 should imply a preference for the other urn in choice 4 , because the change in colors simply reverses winning and losing. Indeed, experimental evidence confirms the suspected violation of subjective expected utility theory (Becker and Brownson, 1964; MacCrimmon and Larsson, 1979). ${ }^{12}$

According to our model, the desire for clarity along with the desire to pay less attention to negative beliefs would cause an individual to bet on the known urn rather than the

\footnotetext{
${ }^{12}$ Non-neutral ambiguity attitudes have been observed in many other experiments as well (e.g., Borghans et al., 2009; Ahn et al., 2013).
} 
ambiguous urn in the Ellsberg paradox. As in Ergin and Gul (2009), uncertainty aversion leads to second-order risk aversion. Proposition 2 from Section 3.3 applies directly to the Ellsberg paradox. Consider $Q_{i}$ to be the question of which ball is drawn from the ambiguously specified urn. Belief about this question depends on the belief about the composition of this urn. On the other hand, belief about $Q_{j}-$ which ball is drawn from the known urn is independent of all other beliefs. ${ }^{13}$

We provide the intuition for this account of the Ellsberg paradox here. When a decision maker is presented with Ellsberg's choices, the following questions, among others, are activated:

- What is the composition of red and black balls in Urn I?

- What is the composition of red and black balls in Urn 2?

Only the second question is known with certainty. Despite having no information from which to form an objective probability over answers to the first question, we assume the decision maker can form a subjective probability (as in Segal (1987) or Seo (2009)), and specifically that the decision maker is likely to believe there to be a uniform distribution over possible compositions of Urn 1. Moreover, savvy decision makers will recognize that payoffs result from a compound lottery with stage one determining the composition of the urn and stage two determining the ball drawn from an urn with that composition, and they will reduce the compound lottery to form a belief that the prize will be won with probability .5. Nevertheless, a bet on a draw from Urn I makes the anticipated payoff contingent on the uncertain answer to the first question, whereas a bet on a draw from Urn II makes the anticipated payoff contingent on the certain answer to the second question.

We rely on three assumptions from Section 2: 1) attention weight on a question increases with the importance of that question; 2) increasing attention weight on a question with an unfavorable belief decreases utility; and 3) uncertain beliefs over answers to which one is indifferent are less favorable than certainty about one such answer. In this case, we assume no preference about the composition of an urn, independent of the eventual payoff, but there is of course the aforementioned preference for certainty (formalized as the one-sided sure-thing principle). We assume that knowing the composition (of Urn II), whatever it may be, is a neutral belief. The belief that Urn I has a uniform distribution over possible compositions, because of this uncertainty, is a negative belief. Thus, the decision

\footnotetext{
${ }^{13}$ The belief that there is a one-half chance of drawing a red ball and a one-half chance of drawing a black ball from the known urn is determined by the belief about its composition, but this belief is held with certainty, and dependence on a probability zero / one event is impossible.
} 
maker prefers not to increase the attention weight on (the composition of) Urn I and can avoid do so by choosing to bet on a draw from Urn II rather than a draw from Urn I. Recognizing informational preference allows us to explain the preference for betting on the known urn rather than on the unknown urn, even when the subjective probability judgment about the odds of winning a prize is the same for both urns. Crucially, our account relies on aversion to missing information rather than a distinction between objective and subjective probabilities. Thus, consistent with Halevy's (2007) experimental findings, we predict that ambiguity preference goes hand in hand with preference over compound (objective) lotteries. ${ }^{14}$

\subsection{Comparative Ignorance Effect}

Note that our explanation of ambiguity preference is inherently context dependent. In the Ellsberg paradox, ambiguity aversion arises from a desire not to pay attention to a salient information gap, combined with the opportunity to shift attention in the desired direction by placing the bet on the known urn. The description of the two urns in comparison makes salient the difference in their composition, so the questions about the composition of the urns get non-negligible attention weight. If, however, an individual is asked to price a bet on a draw from just one of the urns in isolation, the question of the composition of that urn is less salient, and so receives less attention weight. As long as the question is activated, we would expect some degree of ambiguity aversion, because taking a sure payment in lieu of the bet still does shift attention away from an uncertain prospect, but (because attention weight exhibits increasing differences in salience and importance) we would expect the degree of ambiguity aversion to be less when pricing bets on isolated urns than when pricing bets on urns that can be compared. This is precisely the comparative ignorance effect that Fox and Tversky (1995) documented. ${ }^{15}$

Proposition 3 Retain the context of Proposition 2. Consider two possible baseline cognitive states $(\pi, \mathbf{w})$ and $(\pi, \hat{\mathbf{w}})$ that have the same probability judgments but with different attention weights that result from question $Q_{\tilde{\imath}}$ being more salient in the latter state than

\footnotetext{
${ }^{14}$ Ambiguity preference may nevertheless be more extreme than compound lottery preference if the ambiguity makes the uncertainty more salient.

${ }^{15}$ Similarly, if an individual is presented with extraneous information that seems to relate to the ambiguous issue, but is not easily processed, this information activates additional questions about which the individual is uncertain. The individual can shift attention weight away from these uncertain beliefs by avoiding a bet on the ambiguous issue. Indeed, Fox and Weber (2002) find that such unhelpful information makes ambiguous bets appear less attractive.
} 
in the former, i.e., $\hat{\sigma}_{\tilde{\imath}}>\sigma_{\tilde{\imath}}$ and $\hat{\sigma}_{\nu}=\sigma_{\nu}$ for all other $Q_{\nu} \in \mathcal{Q}^{E}{ }^{16}$ A bet attached to question $Q_{i}$ would be more preferable in the former cognitive state than in the latter, i.e., $\mathfrak{u}\left(a_{i} \mid \pi, \mathbf{w}\right)>\mathfrak{u}\left(a_{i} \mid \pi, \hat{\mathbf{w}}\right)$.

Proposition 3 suggests that the comparative ignorance effect is an example of a more salient information gap generating stronger ambiguity aversion. Consistent with this pattern, in a hypothetical scenario involving unknown risks of a vaccine (a scenario that subjects can intuitively grasp), salient missing information about whether the risk was high or had been eliminated made subjects more reluctant to vaccinate than when the subjects faced the same risk presented with no salient missing information (Ritov and Baron, 1990).

Other context effects have been noted as well, ${ }^{17}$ and they may be surprising. Studies have found that ambiguity aversion is exacerbated when others can observe the choice (Curley et al., 1986) and reduced when no others (not even the experimenter) can observe whether the bet wins or loses (Trautmann et al., 2008). The authors interpret this finding to mean that that the preference to avoid subjecting oneself to unknown risks is related to a desire to avoid social disapproval. Our model does not treat social disapproval as a fundamental, but our model could accommodate this phenomenon by positing, plausibly, we believe, that that the possibility of social disapproval makes the unknown composition of the ambiguous urn that much more important if the bet on this urn is chosen.

\subsection{Source Preference}

Context dependence also helps us explain those situations in which ambiguous prospects are, in fact, preferred to risky, but clearly-defined, gambles. In our analysis of the Ellsberg paradox, the prediction of ambiguity aversion depends on shifting attention between single beliefs that all involve neutral answers but that vary in their certainty. In general, shifting attention to favorable issues or away from unfavorable issues should increase utility. That is, we predict a preference for betting on issues one likes thinking about and for not betting on issues one does not like thinking about.

Proposition 4 Retain the context of Proposition 2, but relax the requirement that all answers to question $Q_{\tilde{\imath}}$ have neutral valence. Instead, assume that these answers all have

\footnotetext{
${ }^{16}$ Consider the latter cognitive state to result from joint evaluation of bets attached to questions $Q_{i}$ and $Q_{j}$ and the former cognitive state to result from isolated valuation of a bet attached to question $Q_{i}$.

${ }^{17}$ For an example in the domain of risk, lotteries that are presented with narrow bracketing (and thus, we believe, made more salient) generate stronger risk aversion (Gneezy and Potters, 1997; Bellemare et al., 2005; Haigh and List, 2005; Anagol and Gamble, 2011). Proposition 3 of course implies that the salience of an information gap affects risk preferences as well as ambiguity preferences, in accord with this empirical pattern.
} 
the same valence, but allow this valence to be positive or negative, i.e., $v_{\tilde{\imath}}\left(A_{\tilde{\imath}}\right)=v$ for all $A_{\tilde{\imath}} \in \mathcal{A}_{\tilde{i}}$. Preference for a bet attached to question $Q_{i}$ increases in this valence, i.e., $\mathfrak{u}\left(a_{i} \mid \pi, \mathbf{w}\right)$ increases in $v$. Moreover, for sufficiently high $v$, it becomes preferred to a bet attached to question $Q_{j}$, i.e. $a_{i} \succ a_{j}$.

Proposition 4 implies that ambiguity-seeking behavior arises when information gaps are pleasurable to think about, i.e., in special cases in which outcomes have high valence. For example, ardent sports fans may enjoy betting on the outcome of a game they look forward to watching. They would generally prefer to bet on their home team than on other teams, and especially in comparison to a team their home team is playing against (Babad and Katz, 1991; Morewedge, 2013). Cases of pleasurable information gaps may often coincide with issues about which one has significant expertise. To the extent that people generally enjoy thinking about issues for which they have more expertise and dislike unfamiliar situations, Proposition 4 would account for Heath and Tversky's (1991) findings demonstrating a preference to bet on familiar rather than unfamiliar sources of uncertainty.

Also consistent with our hypothesis that gambling is correlated with the valence of an issue is the fact that people become less willing to hold risky assets after realizing a loss (Imas, 2014), as the painful experience of a loss could make thinking about another risky asset more unpleasant. This realization effect could lead to path dependent risk and ambiguity attitudes. Barberis (2011) suggests that such dynamic changes in ambiguity preference may amplify financial panics that begin with relatively modest declines in asset values.

\subsection{Machina Paradoxes}

Machina (2009) introduced two decision problems for which typical patterns of behavior violate the predictions of most models of choice under ambiguity, including Choquet expected utility, maxmin expected utility, $\alpha$-maxmin, variational preferences, and the smooth model of ambiguity aversion (Baillon et al., 2011). As these paradoxes have been so challenging for models of ambiguity aversion to accommodate, we find it illuminating to show how they are compatible with our model of informational preference.

Machina's "50:51 Example" presents an urn holding 50 balls colored red or yellow (in unknown proportion) and 51 colored black or green (also in unknown proportion). Table 1 displays four bets, showing the payoffs contingent upon the ball drawn. We may take 0 , 101,202 , and 303 to be prizes equally spaced on the utility scale, given one's beliefs. ${ }^{18}$ An

\footnotetext{
${ }^{18}$ Actually eliciting prizes that are equally spaced on the utility scale requires, according to our model,
} 


\begin{tabular}{|c|c|c|c|c|}
\hline \multirow[b]{2}{*}{ Bets } & \multicolumn{2}{|c|}{50 balls } & \multicolumn{2}{|c|}{51 balls } \\
\hline & Red & Yellow & Black & Green \\
\hline$a_{1}$ & 202 & 202 & 101 & 101 \\
\hline$a_{2}$ & 202 & 101 & 202 & 101 \\
\hline $\bar{a}_{3}^{-}$ & 303 & $2 \overline{0} \overline{2}$ & $\overline{10} \overline{1}$ & 0 \\
\hline$a_{4}$ & 303 & 101 & 202 & 0 \\
\hline
\end{tabular}

Table 1: Machina's 50:51 Example.

individual chooses between $a_{1}$ or $a_{2}$, then between $a_{3}$ or $a_{4}$. Both choices involve allocating prizes between yellow and black with the remaining prizes fixed, but the contexts vary in how these remaining prizes are fixed. Bets $a_{2}$ and $a_{4}$ allocate the larger prize to black rather than yellow, which, if the individual accepts the principle of insufficient reason, means greater expected value. Bets $a_{1}$ and $a_{3}$, on the other hand, reduce how much is at stake depending on the unknown proportions in the urn. While they each reduce the stakes by the same absolute amount, bet $a_{1}$ eliminates all dependence on these uncertainties, whereas bet $a_{3}$ does not. The typical preference, $a_{1} \succ a_{2}$ and $a_{3} \prec a_{4}$ (at least when the magnitude of the payoffs is tuned just right), reflects a willingness to forego some material payoffs (in expectation) in order to lessen one's exposure to the unknown when the remaining exposure is minimal, but not when the remaining exposure is significant.

According to our model, choosing a bet affects utility in two ways. It determines the prize distribution corresponding to one's subjective belief about activated questions, thus directly affecting the expected value of the eventual prize. But, additionally, to the extent the distribution of prizes depends on the answers to various activated questions, a bet affects the importance of these questions, which in turn affects the utility derived from one's beliefs about these questions. As with the Ellsberg paradox, it seems reasonable to assume that all possible compositions of the urn (consistent with the known $50: 51$ split) are subjectively judged to be equally likely and that an individual does not care about the actual proportion or about which ball is drawn apart from the corresponding material payoff (i.e., all answers have neutral intrinsic valence). Drawing a black ball is thus subjectively judged to have a $\frac{.5}{101}$ greater chance than a yellow. By construction, this means that bets $a_{2}$ and $a_{4}$ each offer a gain in expected value of .5 over bets $a_{1}$ and $a_{3}$ respectively. On the other hand, bets $a_{1}$ and $a_{3}$ would lessen the importance of questions about the composition of the urn relative to

subjects to consider random distributions of prizes that are independent of their beliefs about activated questions (following from the property of independence across prizes, described in the appendix). There is a leap of faith in believing that subjects do not activate a question concerning which prize they will actually receive. Our analysis is not disturbed, however, if we accept this merely as an approximation. 
bets $a_{2}$ and $a_{4}$ respectively. This would decrease the attention weight on the uncertain belief about the composition of the urn - a negative belief because of the uncertainty. Decreasing the attention weight on a negative belief, of course, increases utility. Our assumptions do not specify precisely how much the attention weight decreases as the stakes are reduced, but it is perfectly reasonable to think that there is diminishing sensitivity of attention weight to how much is at stake corresponding to an uncertain belief. Thus, our model can easily accommodate a greater gain in utility when rendering an uncertainty completely moot than when partially drawing down a higher-stakes exposure (and merely limiting its importance somewhat). This would allow the pattern $a_{1} \succ a_{2}$ and $a_{3} \prec a_{4}$.

Machina's second paradox, the "Reflection Example", involves a similar urn that is now balanced with 50 red or yellow balls and 50 black or green balls. Table 2 displays four bets, showing the payoffs in the case that each kind of ball is drawn. In this example, the prizes do not need to have equal utility increments, and it's fine to think of them as monetary payoffs. Once again, an individual first chooses between $b_{1}$ or $b_{2}$, then between $b_{3}$ or $b_{4}$.

\begin{tabular}{lccccc}
\hline & \multicolumn{2}{c}{50 balls } & & \multicolumn{2}{c}{50 balls } \\
\cline { 2 - 3 } \cline { 5 - 6 } Bets & Red & Yellow & & Black & Green \\
\hline$b_{1}$ & 0 & 50 & & 25 & 25 \\
$b_{2}$ & 0 & 25 & & 50 & 25 \\
$-\bar{b}_{3}-$ & 25 & -50 & - & -55 & - \\
$b_{4}$ & 25 & 25 & & 50 & 0 \\
\hline
\end{tabular}

Table 2: Machina’s Reflection Example.

As in the 50:51 example, both choices involve allocating prizes between yellow and black with the remaining prizes fixed, and the contexts vary in how these remaining prizes are fixed. Bets $b_{1}$ and $b_{3}$ reduce the stakes that depend on the proportion of black to green balls but increase the stakes that depend on the proportion of red to yellow balls, relative to bets $b_{2}$ and $b_{4}$ respectively. Viewed alternatively, bets $b_{1}$ and $b_{4}$ eliminate exposure to one source of uncertainty while amplifying exposure to another, relative to bets $b_{2}$ and $b_{3}$. Empirically, the most common pattern of choices (exhibited by about half of subjects) is $b_{1} \succ b_{2}$ and $b_{3} \prec b_{4}$, with a sizable minority (slightly above a quarter of subjects) choosing the opposite, and relatively few violating reflection symmetry (L'Haridon and Placido, 2010).

An individual who judges all possible compositions of the urn to be equally probable would determine that the expected values of the prizes associated with these four bets are all equal. Thus, according to our model, the choice between bets would hinge on which bet placed less attention weight on uncertain, negative beliefs. Once again, our model does 
not specify precisely how much importance, or, in turn, attention weight, decreases as the stakes associated with an uncertain belief are drawn down, and there could well be heterogeneity across the population, so the model does not rule out any pattern of behavior in this example. Still, from this perspective, the typical pattern of behavior is not surprising. If, as we hypothesized in order to explain the $50: 51$ example, attention weight exhibits diminishing sensitivity to exposure to an uncertain belief, then eliminating a modest exposure entirely would have a greater effect than partially reducing a large exposure by the same amount. By the informational symmetry between the red/yellow composition and the green/black composition, the (negative) value of the (uncertain) belief about each should be equal. Accordingly, a greater reduction in attention weight would lead to a greater increase in utility, regardless of which uncertainty is rendered moot. That is, we would then predict $b_{1} \succ b_{2}$ and $b_{3} \prec b_{4}$. Thus, diminishing sensitivity of attention weight with respect to the stakes associated with an uncertain belief allows our model to accommodate both of Machina's paradoxes.

\section{An Experimental Test of a Key Prediction}

The 'acid' test of a new theory is to generate testable predictions that other theories do not predict, and which have not already been tested. Here, we report such a test of our theory. The key prediction of the theory is that people will be more willing to bet on, and will bet more on, uncertainties that they like to think about. We created a situation likely to produce strong feelings by having pairs of people compete on a two-part intelligence test, with one person winning and one person losing. Both individuals then had the opportunity to bet on whether they did better on the first part of the test than the second and to make the complementary bet that they did better on the second part of the test than the first. Our premise is that people who won the competition would find it more pleasurable to think about the test, so we predicted that winners would be willing to bet more, in total, on the two com-

plementary bets than would losers. Of course, subjects could not be randomly assigned to the conditions of winning or losing the competition, so there could be a selection effect. To rule out a selection effect, we controlled for idiosyncratic risk preferences by also offering subjects a third bet on a random event involving rolls of dice.

We recruited subjects for time slots, deliberately scheduling two subjects for each slot. The subjects were recruited separately (so most did not know one-another) and participated for a show-up fee of $\$ 10$ and the opportunity to win additional money and/or prizes through incentivized choices. 
The two subjects first competed against one-another on a math quiz to win a nonmonetary prize. The math quiz was derived from previous GRE tests. It consisted of 18 questions, divided into two clusters of 9 problems. One cluster consisted of traditional math problems (e.g., if $5 x+32=42 x$, what is the value of $x$ ?), and the other consisted of quantitative comparison problems (e.g., which is greater: $54 \%$ of 360 or 150?). The order of the two clusters was randomly determined, and subjects were given 6 minutes to work on each cluster, with a warning one minute from the end of each 6 minute interval. The warning instructed them that they had a minute left, and encouraged them to guess as needed to give some answer to each question, since there was no penalty for incorrect answers. Upon completion, quizzes were scored immediately. Subjects were informed of their total score on all 18 problems, but, crucially, were not told their score breakdown on each cluster of the quiz. The subject in each pair who received the higher score was given a bonus prize - a succulent plant with a retail value of $\$ 3-\$ 5$.

Subjects were then told that they would be presented with three gambles and were told that only one of them would count, to be determined randomly. Each gamble was presented sequentially, with no preview of what subsequent gambles would consist of. The first gamble was presented as follows:

\section{Gamble 1 depends on your performance on the quiz. Gamble 1 will pay equal to your wager if your score on the quantitative comparison questions is greater than or equal to your score on the problem solving questions.}

Please indicate how much you are willing to wager. You can wager up to half your money (\$5). If you win the bet, then you will get back double the amount you wager. If you lose the bet, then you will lose the amount you wager.

How much do you want to wager?

$\begin{array}{llllll}\text { nothing } & \$ 1 & \$ 2 & \$ 3 & \$ 4 & \$ 5\end{array}$

The second gamble was the complementary bet that paid only if their score on problem solving questions was greater than or equal to that on the quantitative comparisons questions. The third gamble involved two rolls of a ten-sided die. It paid out if the second roll was greater than or equal to the first roll. The amount that subjects wanted to stake on each of these gambles was elicited in the same way as it was for the first (with the knowledge that only one of their choices would count). An exit survey collected their attitudes regarding the task and prize as well as demographic data. 
Subjects were 102 individuals from Pittsburgh area universities (48 males, 54 females, $\left.M_{\text {age }}=24.75\right)$ who were recruited using the Carnegie Mellon University Center for Behavioral and Decision Research Participant Pool. One subject was excluded from the analysis because he achieved a perfect score on the 20 item test, from which he could infer that his score on the two parts would be equal. ${ }^{19}$

The top rows of Table 3 present means and t-tests comparing amounts gambled and selected other variables as a function of whether an individual did or did not win the prize. As hypothesized, individuals bet much more on gamble 1 and the total of gamble 1 and 2 when they won the prize. (They did not bet more on gamble 2, perhaps because of cognitive dissonance after having just chosen a wager on the complementary bet.) As predicted by the theory, and helping to rule out selection effects and mood effects, winning the prize for performance on the math quiz had no significant impact on the amount subjects staked on the third, unrelated, gamble.

\begin{tabular}{|l|l|l|l|l|l|l|l|}
\hline & gamble 1 & gamble 2 & total $(1 \& 2)$ & gamble 3 & $\begin{array}{l}\text { feeling about } \\
\text { performance }\end{array}$ & $\begin{array}{l}\text { performance } \\
\text { relative to } \\
\text { expectation }\end{array}$ & $\begin{array}{l}\text { liking of } \\
\text { prize }\end{array}$ \\
\hline lost & $\$ 1.84$ & $\$ .84$ & $\$ 2.68$ & $\$ 1.38$ & 3.5 & 3.3 & 2.2 \\
\hline won & $\$ 2.92$ & $\$ .96$ & $\$ 3.88$ & $\$ 1.65$ & 5.1 & 4.2 & 2.5 \\
\hline significance & $p=.005$ & $p=.67$ & $p=.004$ & $p=.38$ & $p<.001$ & $p<.001$ & $p=.007$ \\
\hline $\begin{array}{l}\text { correlation } \\
\text { with total } \\
\text { points }\end{array}$ & $\begin{array}{r}r=.24 \\
p=.014\end{array}$ & $\begin{array}{l}r=-.007 \\
p=.95\end{array}$ & $\begin{array}{l}r=.23 \\
p=.02\end{array}$ & $\begin{array}{l}r=-.03 \\
p=.79\end{array}$ & $\begin{array}{l}r=.54 \\
p<.001\end{array}$ & $\begin{array}{l}r=.17 \\
p=.09\end{array}$ & $\begin{array}{l}r=.04 \\
p=.73\end{array}$ \\
\hline
\end{tabular}

Table 3

Not surprisingly, subjects who won felt better about their performance and reported that they performed better than they would have expected to. An unexpected finding was that those who won the prize reported liking it more than those who did not, perhaps indicating denigration of the prize by those who didn't receive it. The bottom row, which presents Pearson correlations between total points earned on the exam and the same variables, shows that amount staked on the first gamble was positively related to total points earned on the exam, and that feeling about performance on the exam was also strongly correlated with actual performance.

Table 4 presents regressions of total amount staked in gambles 1 and 2 on selected variables. Specification 1 simply reiterates that winning the prize increased the amount staked,

\footnotetext{
${ }^{19}$ We have reported all measures, conditions, and data exclusions. A sample size of 100 was initially chosen arbitrarily and then increased to include one additional pair after one subject's data had to be excluded.
} 
by approximately $\$ 1.20$ (out of a total maximum of $\$ 5.00$ ). Specification 2 shows that winning the prize still increases amount gambled, even after controlling for total score (total score is significant if entered by itself; $p<.05$ ). Specification 3 shows that winning the prize is no longer statistically significant (although the magnitude of the point estimate is not reduced much) once feeling about performance on the test is included in the equation. This supports our theory's account of the effect - i.e., that people like to bet on events they feel good about. Specification 4 shows that the effect of winning the prize is still significant after controlling for demographics (age and gender). Females did stake considerably (\$1.10) less on the two gambles than did men, and females did considerably worse on the test than males (not reported: 10.3 correct versus 11.9 correct; $p<.05$ ). Finally, specification 5 uses amount staked on gamble 3 to control for generalized risk-taking. This does not affect the other coefficients substantially, but the coefficient on the new term is large and highly significant, and the adjusted $R^{2}$ of the overall equation more than doubles.

\begin{tabular}{|c|c|c|c|c|c|}
\hline & 1 & 2 & 3 & 4 & 5 \\
\hline Won Prize & $\begin{array}{l}1.2^{* * * *} \\
(.41)\end{array}$ & $\begin{array}{l}.96^{* * *} \\
(.47)\end{array}$ & $\begin{array}{l}.71 \\
(.46)\end{array}$ & $\begin{array}{l}.95^{* *} \\
(.41)\end{array}$ & $\begin{array}{l}.84^{* *} \\
(.37)\end{array}$ \\
\hline Total Score & & $\begin{array}{l}.07 \\
(.06)\end{array}$ & & & \\
\hline Feeling About Performance & & & $\begin{array}{l}.31^{* *} \\
(.15)\end{array}$ & & \\
\hline Female & & & & $\begin{array}{l}-1.1 * * * \\
(.41)\end{array}$ & $\begin{array}{l}-.85^{* * *} \\
(.37)\end{array}$ \\
\hline Age & & & & $\begin{array}{l}.002 \\
(.03)\end{array}$ & $\begin{array}{l}-.01 \\
(.02)\end{array}$ \\
\hline Amount Staked on Gamble 3 & & & & & $\begin{array}{l}.60^{* * * *} \\
(.12)\end{array}$ \\
\hline Constant & $\begin{array}{l}2.7 * * * \\
(.29)\end{array}$ & $\begin{array}{l}3.3 * * * \\
(.64)\end{array}$ & $\begin{array}{l}1.6^{* * *} \\
(.59)\end{array}$ & $\begin{array}{l}3.5 * * * \\
(.79)\end{array}$ & $\begin{array}{l}2.6 * * * \\
(.72)\end{array}$ \\
\hline Adjusted $R^{2}$ & .071 & .072 & .10 & .12 & .30 \\
\hline
\end{tabular}

$N=101$

Standard errors in parentheses

$p<.10, * * p<.05, * * * p<.01$

Table 4: Regression of total amount staked in gambles $1 \& 2$ on selected variables

\section{Conclusion}

Preferences over beliefs (and the attention paid to them) create preferences for or against risky and ambiguous gambles. This information gap account of attitudes toward risk and ambiguity makes sense of low-stakes risk aversion, the difference between comparative 
and non-comparative responses to ambiguity vis a vis risk, and the sensitivity of ambiguity preference to the source of the uncertainty. It is consistent with empirically documented patterns of behavior that have been difficult for other theories to reconcile. We have established the following testable predictions:

H1 Individuals prefer to avoid actuarially fair lotteries that do not involve events that they particularly enjoy thinking about.

H2 Individuals prefer an equivalent simple lottery to a compound lottery that does not involve events that they enjoy thinking about.

H3 Individuals prefer to wager on uncertainties they enjoy thinking about (i.e., that depend on positive beliefs) than on objectively random events, but prefer such random bets to wagers that depend on negative beliefs.

H4 Individuals forced to choose among wagers that depend on negative beliefs prefer to wager on an uncertainty that is less salient.

Timing effects are not part of our formal model, and intuitions about the effects of time delay runs in both directions. From one point of view, it seems intuitive that the costs (or benefits) associated with thinking about negative (or positive) beliefs would scale with the amount of time that an individual spends thinking about them. To the extent the pleasures or pains of focusing on an information gap account for risk and ambiguity preferences, we should then expect that some time delay between exposure to uncertainty (risk or ambiguity) and resolution of that uncertainty would strengthen risk and ambiguity preferences. On the other hand, there is substantial evidence that the feelings associated with uncertainty are strongest right before uncertainty is going to be resolved (van Winden et al., 2011). This suggests that short- and long-term time discounting will dictate whether time delay strengthens or weakens risk and ambiguity preferences. Although we are reluctant to offer any general predictions about the effect of time delays, to the degree that time delay intensifies risk or ambiguity preferences, we would speculate that the effects would be stronger for people who discount the future less.

The primary determinant of risk and ambiguity preference in our model is how people feel when they think about the information they are missing about a gamble. These feelings are likely to be a function of a wide range of factors, including the outcomes, associated probabilities, the vividness of outcomes, the individuals feeling of expertise, any contextual factors (e.g., residual sadness or elation) which affect the individuals emotional reactions, and a variety of individual dispositional factors. Another tenet of our model is that feelings, and hence preferences, should depend on the salience of the missing information - 
the information gap. Salience is, in turn, likely to depend on situational factors, decision framing, and the existence of counterfactuals that highlight the information gap. We have shown that these effects can make sense of a variety of already established empirical effects, and also provided experimental evidence in support of a key, previously untested, prediction. Many other predictions will, we hope, be tested in future empirical research.

\section{Appendix}

\section{Proof of Proposition 1}

Linearity of $v_{X}$ implies that $u_{X}\left(\pi_{X}[b]\right)=u_{X}\left(\pi_{X}[\neg b]\right)$. However, because bet $b$ spreads out the utilities that would result from discovering either heads or tails, it increases $\gamma_{1}$, which implies that $w_{1}[b]>w_{1}[\neg b]$. By the one-sided sure-thing principle, we know that $v_{1}\left(\pi_{1}\right)<0$ (regardless of whether the bet is taken) because the belief about the coin flip is not degenerate (i.e., because it is uncertain). Accepting the bet would increase attention weight on a negative belief and would thus lower utility, so $\neg b \succ b$.

\section{Proof of Proposition 2}

Actions $a_{i}$ and $a_{j}$ determine subjective probability measures $\pi\left[a_{i}\right]$ and $\pi\left[a_{j}\right]$ and attention weight vectors $\mathbf{w}\left[a_{i}\right]$ and $\mathbf{w}\left[a_{j}\right]$ such that:

1. $\pi_{\mathcal{A}}\left[a_{i}\right](\cdot)=\pi_{\mathcal{A}}\left[a_{j}\right](\cdot)$;

2. conditioning on any belief about questions outside of $\mathcal{Q}^{E}$, we have $\pi_{X}\left[a_{i}\right](\cdot)=$ $\pi_{X}\left[a_{j}\right](\cdot)$

3. $w_{i}\left[a_{i}\right]=w_{j}\left[a_{j}\right]$ and $w_{j}\left[a_{i}\right]=w_{i}\left[a_{j}\right]$;

4. for any $\nu$ such that $Q_{\nu} \in \mathcal{Q}^{E}, \nu \neq i, \nu \neq j$, we have $w_{\nu}\left[a_{i}\right] \geq w_{\nu}\left[a_{j}\right]$ with strict inequality for $\nu=\tilde{\imath}$;

5. for any $\nu$ such that $Q_{\nu} \in \mathcal{Q} \backslash \mathcal{Q}^{E}$, we have $w_{\nu}\left[a_{i}\right]=w_{\nu}\left[a_{j}\right]$.

The first condition holds because instrumental actions determine prizes, but not beliefs. The second condition must hold by the assumption that $Q_{i}$ and $Q_{j}$ have the same subjective probabilities. Condition 3 follows from the assumption that $Q_{i}$ and $Q_{j}$ have the same salience together with the observations that the same material importance is given to each question when the corresponding action is taken (because the questions have the same subjective probabilities and the actions attach the same prizes) and that neither question is important when the other action is taken. The crucially important fourth condition applies because only question $Q_{i}$ has dependence on $\mathcal{Q}^{E} \backslash\left\{Q_{i}, Q_{j}\right\}$, so only action $a_{i}$ can increase the importance of these other questions. Lastly, condition 5 holds because questions outside of $\mathcal{Q}^{E}$ are independent of $Q_{i}$ and $Q_{j}$. 
The assumption of independence across prizes applies to the valence of prizes so that for the default belief about questions outside of $\mathcal{Q}^{E}$, valence is equal for the two actions because they create the same subjective distribution over prizes (condition 2).

Because questions $Q_{i}$ and $Q_{j}$ have the same subjective probabilities as well as the same (neutral) valences for all possible answers, it can be shown (using the assumptions of label independence and linearity with respect to attention weights) that the utility cost of an increase in attention weight on one is equal to the utility cost of the same increase in attention weight on the other.

Any uncertain belief about a question in $\mathcal{Q}^{E}$ must be a negative belief because certainty would be a neutral belief and the one-sided sure-thing principle applies. Thus, by the assumption of monotonicity with respect to attention weights, the increase in attention weight on question $Q_{\tilde{\imath}}$ that occurs for action $a_{i}$ (according to condition 4) causes a decrease in utility.

\section{Proof of Proposition 3}

As in Proposition 2, bet $a_{i}$ attached to question $Q_{i}$ makes question $Q_{\tilde{\imath}}$ more important and thus increases the attention weight on a negative belief. By the assumption that attention weight exhibits increasing differences in salience and importance, the decrease in utility due to this effect is worse in cognitive state $(\pi, \hat{\mathbf{w}})$ when question $Q_{\tilde{\imath}}$ is more salient than in cognitive state $(\pi, \mathbf{w})$.

\section{Proof of Proposition 4}

By our construction, utility exhibits increasing differences in the value of a belief and the attention weight on it. For sufficiently high $v$, even an uncertain belief will be a positive belief. In this case, increasing the attention weight on it increases utility, so the bet $a_{i}$ becomes favored relative to $a_{j}$.

\section{References}

Abdellaoui, M., Baillon, A., Placido, L., Wakker, P. (2011). The Rich Domain of Uncertainty: Source Functions and their Experimental Implementation. American Economic Review 101, 695-723.

Abdellaoui, M., Klibanoff, P., Placido, L. (2013). Experiments on Compound Risk in Relation to Simple Risk and to Ambiguity.

Ahn, D., Choi, S., Gale, D., Kariv, S. (2013). Estimating Ambiguity Aversion in a Portfolio Choice Experiment.

Allais, M. (1953). Le Comportement de l'Homme Rationnel devant le Risque: Critique des Postulats et Axiomes de l'Ecole Americaine. Econometrica 21 (4), 503-546.

Anagol, S. Gamble, K. (2011). Does Presenting Investment Results Asset by Asset Lower Risk Taking? University of Pennsylvania Working Paper.

Andreoni, J., Schmidt, T., Sprenger, C. (2014). Measuring Ambiguity Aversion: Experimental Tests of Subjective Expected Utility. In prep.

Anscombe, F., Aumann, R. (1963). A Definition of Subjective Probability. Annals of Mathematical Statistics 34, 199-205. 
Babad, E., Katz, Y. (1991). Wishful Thinking: Against all Odds. Journal of Applied Social Psychology 21, 1921-1938.

Bach, D., Seymour, B., Dolan, R. (2009). Neural Activity Associated with the Passive Prediction of Ambiguity and Risk for Aversive Events. The Journal of Neuroscience 29, 1648-1656.

Baillon, A., L'Haridon, O., Placido, L. (2011). Ambiguity Models and the Machina Paradoxes. American Economic Review 101, 1547-1560.

Barberis, N. (2011). Psychology and the Financial Crisis of 2007-2008.

Becker, S., Brownson, F. (1964). What Price Ambiguity? Or the Role of Ambiguity in Decision-Making. Journal of Political Economy 72, 62-73.

Bellemare, C., Krause, M., Kröger, S., Zhang, C. (2005). Myopic Loss Aversion: Information Feedback vs. Investment Flexibility. Economics Letters 87, 319-324.

Bernasconi, M., Loomes, G. (1992). Failures of the Reduction Principle in an EllbsergType Problem. Theory and Decision 32(1), 77-100.

Bordalo, P., Gennaioli, N., Shleifer, A. (2012). Salience Theory of Choice Under Risk. Quarterly Journal of Economics 127 (3), 1243-1285.

Borghans, L., Heckman, J., Golsteyn, B., Meijer, H. (2009). Gender Differences in Risk Aversion and Ambiguity Aversion. Journal of the European Economic Association 7, 649-658.

Brass, M., von Cramon, D. (2004). Selection for Cognitive Control: A Functional Magnetic Resonance Imaging Study on the Selection of Task-Relevant Information. The Journal of Neuroscience 24, 8847-8852.

Callen, M., Isaqzadeh, M., Long, J., Sprenger, C. (2013). Violence and Risk Preference: Experimental Evidence from Afghanistan? American Economic Review forthcoming.

Caplin, A., Leahy, J. (2001). Psychological Expected Utility Theory And Anticipatory Feelings. Quarterly Journal of Economics 116 (1) 55-79.

Cohn, A., Engelmann, J., Fehr, E., Maréchal, M. (2015). Evidence for Countercyclical Risk Aversion: An Experiment with Financial Professionals. American Economic Review 105 (2).

Curley, S., Yates., F., Abrams, R. (1986). Psychological Sources of Ambiguity Avoidance. Organizational Behavior and Human Decision Processes 38, 230-256.

Diecidue, E., Schmidt, U., Wakker, P. (2004). The Utility of Gambling Reconsidered. Journal of Risk and Uncertainty 29, 241-259.

Ellsberg, D. (1961). Risk, ambiguity, and the Savage axioms. Quarterly Journal of Economics 75, 643-699.

Epstein, L. (2008). Living with Risk. Review of Economic Studies 75 (4), 1121-1141.

Ergin, H., Gul, F. (2009). A theory of subjective compound lotteries. Journal of Economic Theory 144, 899-929.

Fishburn, P. (1980). A Simple Model for the Utility of Gambling. Psychometrika 45, 435-448.

Fox, C., Tverksy, A. 1995. Ambiguity Aversion and Comparative Ignorance. Quarterly Journal of Economics 110 (3), 585-603. 
Fox, C., Weber, M. (2002). Ambiguity Aversion, Comparative Ignorance, and Decision Context. Organizational Behavior and Human Decision Processes 88, 476-498.

Frisch, D., Baron, J. (1988). Ambiguity and rationality. Journal of Behavioral Decision Making 1, 149-157.

Ghirardato, P., Maccheroni, F., Marinacci, M. (2004). Differentiating Ambiguity and Ambiguity Attitude. Journal of Economic Theory 118, 133-173.

Gilboa, I., Schmeidler, D. (1989). Maxmin Expected Utility with Non-Unique Prior. Journal of Mathematical Economics 18 (2), 141-153.

Golman, R., Loewenstein, G. (2015a). An Information-Gap Framework for Capturing Preferences About Uncertainty. Proceedings of the Fifteenth Conference on Theoretical Aspects of Rationality and Knowledge.

Golman, R., Loewenstein, G. (2015b). Curiosity, Information Gaps, and the Utility of Knowledge.

Gneezy, U., List, J., Wu, G. (2006). The Uncertainty Effect: When a Risky Prospect is Valued Less than its Worst Possible Outcome. Quarterly Journal of Economics 121 (4), 1283-1309.

Gneezy, U., Potters, J. (1997). An Experiment on Risk Taking and Evaluation Periods. Quarterly Journal of Economics 112 (2), 631-645.

Haigh, M., List, J. (2005). Do Professional Traders Exhibit Myopic Loss Aversion? An Experimental Analysis. Journal of Finance 60 (1), 523-534.

Halevy, Y. (2007). Ellsberg Revisited: An Experimental Study. Econometrica 75 (2), 503536.

Heath, C., Tversky, A. (1991). Preference and Belief: Ambiguity and Competence in Choice under Uncertainty. Journal of Risk and Uncertainty 4 (1), 5-28.

Holt, C., Laury, S. (2002). Risk Aversion and Incentive Effects. American Economic Review 92 (5), 1644-1655.

Hsee, C., Kunreuther, H. (2000). The Affection Effect in Insurance Decisions. Journal of Risk and Uncertainty 20, 141-159.

Hsu, M., Bhatt, M., Adolphs, R., Tranel, D., Camerer, C. (2005). Neural Systems Responding to Degrees of Uncertainty in Human Decision-Making. Science 310, 1680-1683.

Huettel, S., Stowe, C., Gordon, E., Warner, B., Platt, M. (2006). Neural Signatures of Economic Preferences for Risk and Ambiguity. Neuron 49, 765-775.

Imas, A. (2014). The Realization Effect: Risk Taking after Realized versus Paper Losses. In prep.

Kahneman, D., Tversky, A. (1979). Prospect Theory: An Analysis of Decision Under Risk. Econometrica 47, 263-291.

Keppe, H.-J., Weber, M. (1995). Judged knowledge and ambiguity aversion. Theory and Decision 39, 51-77.

Kilka, M., Weber, M. (2000). Home bias in international stock return expectations. Journal of Psychology and Financial Markets 1, 176-192.

Klibanoff, P., Marinacci, M., Mukerji, S. (2005). A Smooth Model of Decision Making under Ambiguity. Econometrica 73, 1849-1892. 
Kocher, M., Krawczyk, M., van Winden, F. (2014). Let Me Dream On! Anticipatory Emotions and Preference for Timing in Lotteries. Journal of Economic Behavior and Organization 2, 29-40.

Köszegi, B., Rabin, M. (2006). A Model of Reference-Dependent Preferences. Quarterly Journal of Economics 121(4), 1133-1165.

L'Haridon, O., Placido, L. (2010). Betting on Machina's Reflection Example: An Experiment on Ambiguity. Theory and Decision 69 (3), 375-393.

Loewenstein, G., Weber, E., Hsee, C., Welch, N. (2001). Risk as Feelings. Psychological Bulletin 127, 267-286.

Loomes, G., Sugden, R. (1982). Regret Theory: An Alternative Theory of Rational Choice under Uncertainty. Economic Journal 92, 805-824.

Luce, R.D., Raiffa, H. (1957). Games and Decisions. New York: Wiley.

Maccheroni, F., Marinacci, M., Rustichini, A. (2005). Ambiguity Aversion, Robustness, and the Variational Representation of Preferences. Econometrica 74, 1447-1498.

MacCrimmon, K., Larsson, S. (1979). Utility Theory: Axioms Versus "Paradoxes". In Expected Utility Hypotheses and the Allais Paradox, M. Allais and O. Hagen (Eds.), Holland: D. Reidel Publishing Company.

Machina, M. (2009). Risk, Ambiguity, and the Rank-Dependence Axioms. American Economic Review 99 (1), 385-392.

Morewedge, C. (2013). Reluctance to Hedge. In prep.

Nau, R. (2006). Uncertainty Aversion with Second-Order Utilities and Probabilities. Management Science 52, 136-145.

Prelec, D., Loewenstein, G. (1998). The Red and the Black: Mental Accounting of Savings and Debt. Marketing Science 17, 4-28.

Quiggin, J. (1982). A Theory of Anticipated Utility. Journal of Economic Behavior and Organization 3 (4), 323-343.

Rabin, M. (2000). Risk Aversion and Expected-utility Theory: A Calibration Theorem. Econometrica 68 (5), 1281-1292.

Ritov, I., Baron, J. (1990). Reluctance to vaccinate: Omission bias and ambiguity. Journal of Behavioral Decision Making 3 (4), 263-277.

Savage, L. (1954). The Foundations of Statistics. New York: Wiley.

Schmeidler, D. (1989). Subjective Probability and Expected Utility without Additivity. Econometrica 57 (3), 571-587.

Schmidt, U., Starmer, C., Sugden, R. (2008). Third-Generation Prospect Theory. Journal of Risk and Uncertainty 36, 203-223.

Segal, U. (1987). The Ellsberg Paradox and Risk: An Anticipated Utility Approach. International Economic Review 28, 175-202.

Segal, U. (1990). Two-Stage Lotteries without the Reduction Axiom. Econometrica 58, 349-377.

Seo, K. (2009). Ambiguity and Second-Order Belief. Econometrica 77, 1575-1605.

Simonsohn, U. (2009). Direct Risk Aversion. Psychological Science 20 (6), 686-692. 
Spears, D. (2013). Poverty and Probability: Aspiration and Aversion to Compound Lotteries in El Salvador and India. Experimental Economics 16 (3), 263-284.

Taylor, K. (1995). Testing credit and blame attributions as explanation for choices under ambiguity. Organizational Behavior and Human Decision Processes 54, 128-137.

Taylor, S., Brown, J. (1988). Illusion and Well-Being: A Social Psychological Perspective on Mental Health. Psychological Bulletin 103 (2), 193-210.

Trautmann, S., Vieider, F., Wakker, P. (2008). Causes of ambiguity aversion: Known versus unknown preferences. Journal of Risk and Uncertainty 36, 225-243.

Tversky, A., Kahneman, D. (1992). Advances in Prospect Theory: Cumulative Representation of Uncertainty. Journal of Risk and Uncertainty 5, 297-323.

Tymula, A., Belmaker, L., Roy, A., Ruderman, L., Manson, K., Glimcher, P., Levy, I. (2012). Adolescents' Risk-Taking Behavior Is Driven by Tolerance to Ambiguity. Proceedings of the National Academy of Sciences 109 (42), 17135-17140.

van Winden, F., Krawczyk, M., Hopfensitz, A. (2011). Investment, Resolution of Risk, and the Role of Affect. Journal of Economic Psychology 32 (6), 918-939.

von Neumann, J., Morgenstern, O. (1944). Theory of Games and Economic Behavior. Princeton: Princeton University Press.

Weaver, R., Frederick, S. (2012). A Reference Price Theory of the Endowment Effect. Journal of Marketing Research, forthcoming.

Weinstein, N. (1980). Unrealistic Optimism About Future Life Events. Journal of Personality and Social Psychology 39 (5), 806-820.

Yang, Y., Vosgerau, J., Loewenstein, G. (2013). The Influence of Framing on Willingness to Pay: An Explanation for the Uncertainty Effect. 